patients harboring non-ruptured saccular or fusiform intracranial aneurysms with a wide neck $(>4 \mathrm{~mm})$ located in the anterior or posterior circulation. Standard dual anti-platelet therapy was started 5 days pre-procedure and continued for at least 6 months. Neurological complications were classified as intra-procedural, early or delayed if they arose within 1, 30 or more than 30 days from treatment, respectively. Neurological complications were considered minor and major if resulting in transient or permanent neurological morbidity, respectively. Clinical status at discharge and follow up was assessed by independent neurologists or neurosurgeons and imaging included MRI angiography (MRA), CT angiography (CTA) and DSA, depending on the institution.

Results The study included 28 patients (23 females, 82\%; age range: 36-86, median age: 56) treated between April and November 2019, harboring 29 aneurysms. Twenty-eight aneurysms were saccular and in the anterior circulation. The majority of aneurysms were $<12 \mathrm{~mm}$ in diameter (18/29, 62\%), 8/ 29 (28\%) were large $(<25 \mathrm{~mm}), 2 / 29(7 \%)$ were giant $(>25$ $\mathrm{mm}$ ) and one was fusiform and partially thrombosed (3\%). The SE was delivered in all cases via a tri-axial approach with an intermediate catheter and a 0.027' microcatheter for implant delivery. This low-profile catheter allowed delivery of the implant through the trans-radial route. Thirty-two implants were used ( 4 in the patient with the fusiform aneurysm) with an average of 1.1 stents/patient. There were no intra-procedural complications. Median clinical and imaging follow up time was 6 months (range: 2-6 months). There were no deaths. Three patients $(3 / 28,11 \%)$ had early minor neurological complications. There was one (4\%) early major neurological complication in a patient with a hemispheric stroke on post-op day 4. Two patients had minor delayed neurological complications $(2 / 28,7 \%)$. Complete thrombosis was seen in $16 / 29$ aneurysms (55\%, most of those imaged at 6 months), while partial thrombosis was seen in $12 / 29$ aneurysms (41\%). Covered side branches were patent in all patient but the one who experienced the stroke.

Conclusion The SE shows excellent navigability while keeping a high flow-diverting effect. Mid-term clinico-radiological results show good efficacy and acceptable safety of the implant.

Disclosures E. Orru: None. H. Rice: 2; C; Medtronic, Stryker, Philips, Penumbra, Microvention. L. De Villiers: None. A. Wakhloo: 1; C; Philips. 2; C; Stryker, Phenox. 3; C; SCENT trial. 4; C; InNeuroCo, EpiEP, Neural Analytics, Rist, Analytics 4 Life, ThrombX. G. Song Chia: None. A. Qureshi: None. T. Krings: 2; C; Stryker, Medtronic, Penumbra. 4; C; Marblehead Inc. V. Pereira: 1; C; Philips Healthcare. 2; C; Stryker, Balt, Cerenovous, Medtronic.

\section{E-233 RUPTURED INTRACRANIAL ANEURYSMS TREATED WITH THE PIPELINE EMBOLIZATION DEVICE: A SYSTEMATIC REVIEW AND POOLED ANALYSIS OF INDIVIDUAL PATIENT DATA}

${ }^{1} \mathrm{P}$ Foreman, ${ }^{2} \mathrm{~A}$ llyas*, ${ }^{1} \mathrm{M}$ Cress, ${ }^{1} \mathrm{~J}$ Vachhani, ${ }^{1} \mathrm{R}$ Hirschl, ${ }^{3} \mathrm{C}$ Griessenauer. ${ }^{1}$ Neurosurgery, Orlando Health, Orlando, $\mathrm{FL}_{;}{ }^{2}$ Neurosurgery, University of Alabama at Birmingham, Birmingham, $A L ;{ }^{3}$ Neurosurgery, Geisinger Health System, Danville, PA

\subsection{6/neurintsurg-2020-SNIS.264}

Objectives The pipeline embolization device (PED) is a flow diverting stent used for the treatment of intracranial aneurysms. This technique necessitates dual antiplatelet therapy
(DAPT) and aneurysm occlusion is delayed. Despite these limitations, the PED is used for a subset of ruptured aneurysms not amendable to traditional treatment techniques. We seek to evaluate the safety and efficacy of this practice.

Methods A systematic review of the PubMed and MEDLINE databases from January of 2011 to October of 2019 was performed to identify studies of ruptured intracranial aneurysms treated with the PED. Inclusion required publication in English, a minimum of 5 cases and reporting of clinical outcome and complications. Duplicate patient reports from the same author(s) were excluded. Individual patient data was collected for pooled analysis.

Results Individual patient data was collected for 140 patients with 140 ruptured aneurysms treated with the PED from 11 studies. Seventy-four $(73.3 \%)$ out of 101 patients were female and the mean age 50.9 years. Hunt and Hess score was 1-3 for $84.2 \%$ of patients. Mean aneurysm size was $6.0 \mathrm{~mm}$ and the majority were blister $(51.0 \%)$ or dissecting $(26.9 \%)$ in morphology. Aneurysms re-hemorrhaged following PED placement in $3(2.1 \%)$ cases: $3 \mathrm{~mm}$ saccular ICA aneurysm, 21 $\mathrm{mm}$ saccular ICA aneurysm, and $34 \mathrm{~mm}$ fusiform ICA aneurysm. Larger aneurysm size $(p=0.05)$ and saccular morphology $(\mathrm{p}=0.01)$ were associated with aneurysm re-hemorrhage. Of the 125 patients with radiographic follow up, 104 (83.2\%) had complete aneurysm occlusion. Smaller aneurysm size was associated with complete aneurysm occlusion $(p=0.03)$. Symptomatic neurologic complications occurred in 18 (12.4\%) and symptomatic neurologic complications were associated with increasing Hunt and Hess score and Fisher grade.

Conclusion The majority of ruptured aneurysms treated with the PED and reported in the literature were blister or dissecting in morphology. Treatment of ruptured aneurysms was associated with a re-hemorrhage rate of $2.1 \%$ and complete occlusion rate of $83.2 \%$. Flow diversion with the PED is a viable option for ruptured aneurysms not amendable to traditional surgical and endovascular treatment options.

Disclosures P. Foreman: None. A. Ilyas: None. M. Cress: None. J. Vachhani: None. R. Hirschl: None. C. Griessenauer: None.

\section{E-234 SINGLE-CENTRE STUDY OF ENDOVASCULAR TREATMENT OF MIDDLE CEREBRAL ARTERY (MCA) ANEURYSMS}

${ }^{1} \mathrm{G}$ Chia*, ${ }^{1} \mathrm{H}$ Rice, ${ }^{1} \mathrm{M}$ Jaya Kumar, ${ }^{1} \mathrm{R}$ Sharma, ${ }^{1} \mathrm{C}$ Rapier, ${ }^{2} \mathrm{~T}$ Withers, ${ }^{1} \mathrm{~L}$ de Villiers. ${ }^{1}$ Radiology, Gold Coast University Hospital, Southport, Queensland, AUSTRALIA; ${ }^{2}$ Neurosurgery, Gold Coast University Hospital, Southport, Queensland, Australia

\subsection{6/neurintsurg-2020-SNIS.265}

Purpose To evaluate safety and efficacy of endovascular treatment of MCA aneurysms. Current published data does not clearly demonstrate an advantage with either endovascular treatment or open neurosurgical clipping.

Methods and Materials Consecutive patients with ruptured and unruptured MCA aneurysms, treated with endovascular methods in our comprehensive multidisciplinary institution, from January 2008 to September 2019 were included. Database was prospectively acquired and retrospectively analysed. Baseline patient demographics, aneurysm location, size and previous treatments were collected. Imaging follow up was performed at 3 months, 6 months, 18 months, 2 years and 5 years. Data analyses focused on type of device used, procedure-related 
adverse events, long term angiographic results and clinical outcomes.

Results 140 treatments on 124 MCA aneurysms were performed in 111 patients (18 M1, 91 M1/2 bifurcation, 11 M2, 4 M3 or M4 aneurysms). 45\% treated were ruptured aneurysms. 66 were treated with coils alone, 5 with stent-assisted coiling, 19 with a combination of coils and flow diverting stents, 47 with flow diverting stents alone, and 3 with WEB devices. Occlusion rates were $81 \%, 90 \%$ and $88 \%$ at 6 months, 2 years and 5 years respectively. Specifically, occlusion rates for aneurysms treated with Pipeline flow diverting stents were $92 \%$ at 6 months and $100 \%$ at 1 year with no subsequent recurrence. All cause procedure-related morbidity was $3.6 \%(5 / 140)$ and mortality $1.4 \%(2 / 140)$.

Conclusion Endovascular treatment of MCA aneurysms results in high rates of complete aneurysm occlusion with low rates of complication. Treatment with flow diverting stents have resulted in high durable aneurysm occlusion rates.

Disclosures G. Chia: None. H. Rice: 1; C; Medtronic, Stryker, Microvention, Philips, Siemens and GE. 2; C; Medtronic, Stryker, Microvention, Philips, Siemens and GE. M. Jaya Kumar: None. R. Sharma: None. C. Rapier: None. T. Withers: None. L. de Villiers: 1; C; Medtronic, Stryker, Microvention, Philips, Siemens and GE. 2; C; Medtronic, Stryker.

\section{E-235 USE OF 45 MG TWICE DAILY TICAGRELOR WITH 81 MG ASPIRIN FOR ENDOVASCULAR TREATMENT OF UNRUPTURED INTRACRANIAL ANEURYSMS: A SINGLE CENTER EXPERIENCE}

T Mehta, K Masood, 55414*, M Ghannnam, 55414, A Grande, 55414, R Tummala, 55414, B Jagadeesan, 55414. Neurology, Neurosurgery and Radiology, University of Minnesota, Minneapolis, MN

\subsection{6/neurintsurg-2020-SNIS.266}

Introduction Stent assisted coiling (SAC) and Flow diverter (FD) placement are commonly used techniques for the treatment of unruptured intracranial aneurysms (UIA). Stents and FDs require dual antiplatelet therapy (DAPT) to prevent instent thrombosis. The selection of medications is mainly operator dependent. Cardiovascular literature suggests that the use of Aspirin $81 \mathrm{mg}$ in conjunction with low dose Ticagrelor (45 $\mathrm{mg}$ twice daily) is superior than the use of Clopidogrel $75 \mathrm{mg}$ and Aspiring $325 \mathrm{mg}$.

Methods We performed a retrospective chart review of patients who underwent treatment of UIA at our institution using SAC or FD from 2016-2019. The patients who are on anticoagulation or did not have a pre-procedure P2Y12 inhibition assay were excluded. The patient group receiving Brilinta $45 \mathrm{mg}$ twice daily with aspirin $81 \mathrm{mg}$ daily (Group 1) was compared with the patient group receiving Plavix $75 \mathrm{mg}$ daily with aspirin $325 \mathrm{mg}$ daily (Group 2). The primary outcome was defined as intraoperative or acute (during hospitalization) postoperative stroke. Secondary outcomes included ischemic stroke after discharge within one year from treatment, and differences in P2Y12 inhibition assay values between the two groups.

Results A total of 111 patients were identified that met the inclusion criteria, $42(37.8 \%)$ in Group 1, and 69 in Group 2 $(62.2 \%)$ protocol. There was no significant difference in the primary outcome between the two groups; Group 1, (2 patients,4.7\%), and Group 2 (1 patient,1.4\%) $(p=0.11)$. All the primary end point events in both groups were related to
FD. Clinically significant ischemic stroke after discharge within one year was also not significantly different (2 patients $(4.7 \%)$ in Group 1 Vs. 3 patients (4.3\%) in Group 2, p=0.277). The P2Y12 inhibition assay level was significantly lower in Group 1, when compared to Group 2 (71.6 Vs 94.8, $\mathrm{p}=0.027$ ). There was no significant difference in the number of patients achieving a therapeutic value for the P2Y12 inhibition assay value ( $<194$ at our instituion's laboratory) (83.3 in Group 1 Vs. 82.6\%, $\mathrm{p}=0.92)$. One patient in Group 2 experienced a clinically significant groin hematoma, and one patient in the same group experienced a significant gastrointestinal bleed. No significant hemorrhagic events occured in patients belonging to Group 2. There was also no instance of remote intracerebral hemorrhage or post treatment aneurysm rupture in either group.

Conclusion Lower preoperative P2Y12 assay values from the use of $45 \mathrm{mg}$ twice daily Ticagrelor with $81 \mathrm{mg}$ Aspirin for treatment of UIAs with SAC or FD placement did not result in decreased rates of perioperative stroke or delayed stroke rates within 1 year of surgery when compared to the well established use of Clopidogrel $75 \mathrm{mg}$ and Aspirin $325 \mathrm{mg}$.

Disclosures T. Mehta: None. K. Masood: None. M. Ghannnam: None. A. Grande: None. R. Tummala: None. B. Jagadeesan: None.

\section{E-236 TECHNICAL ASPECTS OF COMBINED INTRASACCULAR AND ENDOLUMINAL FLOW DIVERSION}

T White*, K Shah, J Turpin, J Katz, H Woo. Neurosurgery, Northwell Health, Manhasset, NY

\subsection{6/neurintsurg-2020-SNIS.267}

Introduction The concurrent use of the Pipeline Embolization Device (PED) with coils has been shown to be beneficial when treating complex or large aneurysms. Alternatives to coiling as an adjunctive treatment are currently limited. The FDA recently approved the Woven EndoBridge (WEB) (Microvention, Aliso Viejo, California) device as an intrasaccular flow diverter for wide neck bifurcation aneurysms (FDA). Here in we present the technical aspects of combined WEB plus PED for the treatment of intracranial aneurysms.

Methods A retrospective chart review of all patients treated via intrasaccular flow diversion at a single institution over the last 12 months was done. In total 34 aneurysms were treated via WEB intrasaccular flow diversion; however, only 7 of these aneurysms were treated with the pipeline embolization device as well. Technical aspects of each procedure were recorded.

Results In total 6 patients underwent the treatment of 7 aneurysms via combined intrasaccular and endoluminal flow diversion. All aneurysm were treated in a single setting but for one patient. This patient came in with subarachnoid hemorrhage, had a WEB placed, and subsequently was found to have recanalized their aneurysm due to WEB compaction. This prompted placement of the a Pipeline 4 weeks after initial treatment. There were no complications associated with any treatment. Post embolization MRI/A showed no new infarcts in $3 / 7$ patients and small punctate DWI changes in $4 /$ 7 patients with good stent flow in all cases. There were no bifurcation aneurysms: 5 posterior communicating, 1 superior hypophyseal, 1 vertebrobasilar. The majority of the aneurysms required steam shaping of the Via deployment catheter in order to place the WEB orthogonally to the aneurysm dome. 